\title{
Dexmedetomidine alleviates diabetic neuropathic pain by inhibiting microglial activation via regulation of $\mathrm{miR}$ - 618/P2Y12 pathway
}

\author{
Jiannan Song ${ }^{1}$, Shan Cong ${ }^{1}$, Yan Qiao ${ }^{2 *}$ \\ ${ }^{1}$ Department of Anesthesiology, Chifeng Municipal Hospital, ${ }^{2}$ Department of Neurology, Chifeng Municipal Hospital, Chifeng \\ City, Inner Mongolia Autonomous Region, 024000, China
}

*For correspondence: Email: qiaoyan12300@163.com; Tel: +86-0476-8365914

Sent for review: 13 October 2020

Revised accepted: 27 December 2020

\begin{abstract}
Purpose: To investigate the effect of dexmedetomidine on streptozotocin (STZ)-induced diabetic neuropathy pain (DNP) in rats and elucidate its mechanism of action.

Methods: The DNP rat model was established by injecting STZ (70 mg/kg) following dexmedetomidine treatment. Next BV-2 cells were stimulated using lipopolysaccharide (LPS, $200 \mathrm{ng} / \mathrm{mL}$ ) and then administered $20 \mu \mathrm{M}$ dexmedetomidine. Blood glucose levels, body weight, and paw withdrawal threshold (PWT) were measured once a week in DNP rats. Transfection was performed, and luciferase reporter assay was used to verify microRNA (miR)-337 binding to Rap1A mRNA. Reverse transcriptionpolymerase chain reaction (RT-PCR) was used to measure the levels of miR-618 and P2Y12 while the protein levels of P2Y12 and ionized calcium-binding adaptor molecule 1 (IBA-1) were determined by western blot analysis.

Results: Dexmedetomidine treatment significantly increased PWT $(p<0.01)$ in DNP rats and decreased miR-618 expression $(p<0.01)$ but increased P2Y12 expression $(p<0.01)$ in the spinal cord of DNP rats. Luciferase reporter assay data showed that the presumed binding site of miR-618 is located in the 3'-untranslated regions of P2Y12. MiR-618 overexpression significantly reduced P2Y12 levels $(p<0.01)$. Dexmedetomidine upregulated P2Y12 expression $(p<0.01)$ but decreased IBA-1 expression $(p<0.01)$.

Conclusion: Dexmedetomidine application attenuates DNP by inhibiting microglial activation via the regulation of miR-618/P2Y12 pathway. This finding provides a potential therapeutic strategy for DNP management.
\end{abstract}

Keywords: Dexmedetomidine, Diabetic neuropathy pain, Paw withdrawal threshold, Calcium-binding adaptor molecule 1, MiR-618, P2Y12

\begin{abstract}
This is an Open Access article that uses a fund-ing model which does not charge readers or their institutions for access and distributed under the terms of the Creative Commons Attribution License (http://creativecommons.org/licenses/by/4.0) and the Budapest Open Access Initiative (http://www.budapestopenaccessinitiative.org/read), which permit unrestricted use, distribution, and reproduction in any medium, provided the original work is properly credited.
\end{abstract}

Tropical Journal of Pharmaceutical Research is indexed by Science Citation Index (SciSearch), Scopus, International Pharmaceutical Abstract, Chemical Abstracts, Embase, Index Copernicus, EBSCO, African Index Medicus, JournalSeek, Journal Citation Reports/Science Edition, Directory of Open Access Journals (DOAJ), African Journal Online, Bioline International, Open-J-Gate and Pharmacy Abstracts

\section{INTRODUCTION}

Diabetic neuropathy pain (DNP) is one of the most common symptoms in patients with diabetes mellitus [1]. Approximately $30 \%$ of patients develop neuropathic pain, depression, anxiety, and sleep deprivation, which have serious impacts on the quality of life and lead to 
an increased economic burden for diabetes care $[1,2]$. The activation of microglia in the spinal cord is necessary for pain hypersensitivity after nerve injury, and inhibiting microglia may provide a potential therapeutic strategy [3]. Thus, it is of great importance to regulate the activity of microglia in patients with DNP.

Dexmedetomidine, a potent alpha-2-adrenergic agonist, has neuroprotective activities, such as reducing delirium, maintaining sleep architecture and ventilatory drive, and decreasing sympathetic tone and the inflammatory response [4]. A recent study unveiled that dexmedetomidine attenuates neuroinflammation by regulating the polarization of the microglial pro-inflammatory and anti-inflammatory status [5]. Dexmedetomidine alleviated neuropathic pain after spared nerve injury by repressing microglia activity via suppressing spinal P2X4 receptors, phosphorylated p38, and brain-derived neurotrophic factor in the spinal dorsal horn [6]. In addition, dexmedetomidine attenuated DNP in rats by regulating the Wnt $10 a / \beta$-catenin pathway [7]. However, the effect of dexmedetomidine on microglia activity in DNP and its underlying mechanism remain poorly understood and requires further study. A growing number of studies have shown that microRNAs (miRNAs) play important roles in regulating many diseases $[8,9]$ Inhibiting miR-618 promotes osteoclastogenesis, which plays a pivotal role in postmenopausal osteoporosis [10]. miR-618 also inhibits metastasis in gastric cancer by decreasing transforming growth factor $\beta 2$ expression [11]. In addition, miR-618 was elevated in type 1 diabetes patients with many microvascular complications [12]. However, the role of miR-618 in DNP remains unclear. Thus, the relationship between dexmedetomidine and miR-618 in DNP warrants in-depth analysis.

In this study, a DNP rat model was established by injecting STZ, and BV-2 cells were stimulated by LPS. The potential mechanism of dexmedetomidine against DNP was further investigated by focusing on microglia and cell signaling.

\section{EXPERIMENTAL}

\section{Induction of the diabetic neuropathy pain rat} model

Eighteen male Sprague-Dawley rats (weighing $250 \pm 20 \mathrm{~g}$ ) were purchased from Nanjing Junke Bioengineering Co., Ltd (Nanjing, China) and were kept at $22 \pm 2{ }^{\circ} \mathrm{C}$ under a 12-h light-dark cycle. The procedures were approved by the Ethics Committee of Chifeng Municipal Hospital (approval no. 2020-026) and conducted in accordance with the National Institutes of Health Laboratory Animal Care and Use Guidelines [13].

The DNP rat model was established by the intraperitoneal injection of streptozotocin (STZ, $70 \mathrm{mg} / \mathrm{kg}$, Sigma-Aldrich, St. Louis, MO, USA) 3 times/day. Eight days after STZ injection, glucometer IME-DC (Bayer, Leverkusen, Germany) was used to determine the blood glucose levels using blood samples collected from the tail veins, and blood glucose levels $\geq 16$ $\mathrm{mM}$ were considered diabetic. Neuropathic pain in diabetic rats was defined as $50 \%$ PWT $<5 \mathrm{~g}$, measured using the von Frey hair test.

\section{Treatment}

Eight days after STZ injection, the rats were intraperitoneally injected with $50 \mu \mathrm{g} / \mathrm{kg}$ of dexmedetomidine (Yuanye Biological Technology Co. Ltd, Shanghai, China) once a day for 34 days. The animals in the control group were injected with an equivalent volume of saline. The blood glucose levels were determined using a glucometer IME-DC (Bayer), and the body weights were recorded once a week after STZ injection until the end of the experiments. In addition, the von Frey hair test, which was used to measure the paw withdrawal threshold (PWT), was performed once a week.

For miR-618 and P2Y12 expression in the spinal cord of rats, different concentrations of dexmedetomidine $(5,10,50$, and $100 \mu \mathrm{g} / \mathrm{kg})$ were administered. On the last day of the experiment, the rats were sacrificed under general anesthesia with pentobarbital sodium (50 $\mathrm{mg} / \mathrm{kg}$ ). The L4-L6 segments of the spinal cord of rats were isolated immediately and stored in liquid nitrogen.

\section{Cell culture}

BV-2 cells were obtained from the Cell Bank of Chinese Academy of Medical Sciences (Beijing, China) and were kept in Dulbecco's Modified Eagle Medium (Gibco, Grand Island, NY, USA) containing $10 \%$ fetal bovine serum (Gibco) in a 5 $\% \quad \mathrm{CO}_{2}$ incubator at $37{ }^{\circ} \mathrm{C}$. Cells $\left(1 \times 10^{5}\right.$ cells/well) were cultured in 24-well plates overnight. Next, the cells were stimulated using $200 \mathrm{ng} / \mathrm{mL}$ of LPS, followed by treatment with 20 $\mu \mathrm{M}$ dexmedetomidine or shP2Y12 for $24 \mathrm{~h}$ and western blot analysis.

\section{MiRNA transfection}

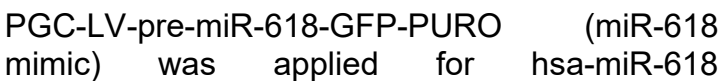

Trop J Pharm Res, January 2021; 20(1): 62 
overexpression, and GC-LV-miR-NC-GFP-PURO (mimic control) was used as a control for miR618 mimic. Cell transfections were performed using Lipofectamine 3000 reagent (Invitrogen, Carlsbad, CA, USA). HEK293 cells were then transfected with miR-618 mimic and NC mimic.

\section{Luciferase report assays}

After $48 \mathrm{~h}$ of transfection, cells were collected and cell lysates were obtained. A dual-reporter luciferase assay system was utilized to determine luciferase activity using a Tecan SpectraFluorPlus plate-reader (Plastic Surgery Laboratory, University of Pittsburgh, Pittsburgh, PA, USA). Renilla luciferase was used for normalization.

\section{Reverse transcription-polymerase chain reaction (RT-PCR)}

The total RNA of BV-2 and HEK293 cells was isolated using TRIzol reagent (Invitrogen). Firststrand cDNA was synthesized using the SuperScript@ III cDNA Synthesis Kit (Invitrogen). The QuantiTect SYBR Green PCR kit (Qiagen, Hilden, Germany) and Icycler iQ Multi-color realtime PCR detection system were used for RT$\mathrm{PCR}$. The primer pairs used for amplification are shown in Table 1. Reverse transcriptionpolymerase chain reaction was performed as follows: $95{ }^{\circ} \mathrm{C}$ for $10 \mathrm{~min}, 95{ }^{\circ} \mathrm{C}$ for $5 \mathrm{~s}, 60{ }^{\circ} \mathrm{C}$ for $30 \mathrm{~s}$, and $72{ }^{\circ} \mathrm{C}$ for $30 \mathrm{~s}, 40$ cycles in total. The relative expression levels were quantified using the $2^{-} \Delta \Delta$ CT method [14].

\section{Western blot analysis}

The proteins were extracted from BV-2 and HEK293 cells using lysis buffer (Beyotime, Shanghai, China) and then were quantified using the BCA Protein Assay Kit (Thermo Fisher Scientific, Waltham, MA, USA). Equal amounts of protein were separated by $10 \%$ SDS-PAGE, followed by transfer to nitrocellulose membranes. Next, the membranes were incubated with the primary antibodies P2Y12 (1:1000; AnaSpec Inc., Fremont, CA, USA) and IBA-1 (1:1000; Wako, Osaka, Japan) overnight at $4{ }^{\circ} \mathrm{C}$. Glyceraldehyde-3-phosphate dehydrogenase (GAPDH; 1:1000, Abcam, Cambridge, MA, USA) was used as a loading control. The membranes were washed using TBST $(0.2 \%$ Tween-20 in blocking buffer) and incubated with horseradish peroxidase-conjugated anti-rabbit or anti-mouse IgG (Promega Corporation, Madison, WI, USA). An enhanced chemiluminescence detection system was applied to quantify the intensity of the bands.

\section{Statistical analysis}

SPSS (Version 17.0) (SPSS Inc, Chicago, IL, USA) was utilized for all statistical analyses. The experimental data were reported as means \pm standard deviation. One-way analysis of variance was used to compare multiple groups, followed by Tukey's multiple comparison tests. The statistical significance was $p<0.05$.

\section{RESULTS}

\section{Dexmedetomidine alleviates neuropathic pain} in diabetic rats

The blood glucose of DNP rats was significantly increased within 1 week $(p<0.01)$, and hyperglycemia was maintained during the experiment (Figure $1 \mathrm{~A}$ ). In addition, the body weights were significantly decreased in DNP rats compared with those in the control group (Figure $1 \mathrm{~B} ; p<0.01)$. However, dexmedetomidine administration did not significantly affect the blood glucose level and body weight. PWT was decreased in DNP rats compared with that in the control group $(p<0.01)$, while dexmedetomidine treatment significantly elevated PWT $(p<0.01)$ (Figure $1 \mathrm{C}$ ).

\section{Dexmedetomidine decreases the expression of miR-618 but increases the level of P2Y12 in the spinal cord of DNP rats}

The changes in the expression of miR-618 and P2Y12 in the spinal cord were measured after the administration of different concentrations of dexmedetomidine. STZ stimulation significantly inhibited the expression of P2Y12 mRNA, but the level of miR-618 was increased compared with control group $(p<0.01)$.

Table 1: Primer pairs used for amplification

\begin{tabular}{lll}
\hline Gene & \multicolumn{1}{c}{ Primer pairs } \\
\hline miR-618 & 3'-AAACTCTACTTGTCCTTCTGAGT-5' \\
P2Y12 & 5'-CATTGCTGTACACCGTCCTG-3' (forward) & $\begin{array}{l}\text { 5'-GGCTCCCAGTTTAGCATCAC-3' } \\
\text { (reverse) }\end{array}$ \\
GAPDH & $\begin{array}{l}\text { 5'-CGGCTACCACATCCAAGGAA-3' } \\
\text { (forward) }\end{array}$ & $\begin{array}{l}\text { 5'-AGCCACATCGCTCAGACACC-3' } \\
\text { (reverse) }\end{array}$ \\
\hline
\end{tabular}




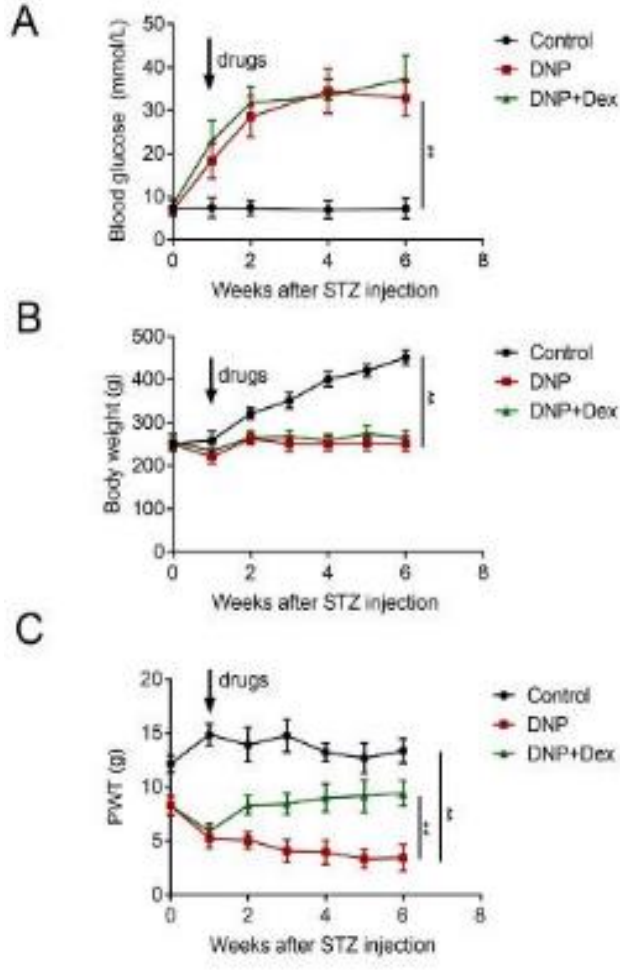

Figure 1: Dexmedetomidine attenuates neuropathic pain in diabetic rats. A: Blood glucose level changes in DNP rats after dexmedetomidine treatment. B: Body weight changes in DNP rats after dexmedetomidine treatment. C, Time course of PWT in DNP rats after dexmedetomidine administration. ${ }^{* *} p<0.01$, compared with the control group or DNP+Dex group. DEX, dexmedetomidine; DNP, diabetic neuropathic pain

Dexmedetomidine administration remarkably increased P2Y12 expression but decreased miR618 expression in the spinal cord (Figure $2 \mathrm{~A}$ and $\mathrm{B} ; p<0.01)$. Further results found the P2Y12 protein level was distinctly decreased in DNP rats, whereas dexmedetomidine elevated P2Y12 expression compared with that in untreated DNP rats (Figure $2 \mathrm{C} ; p<0.01$ ).

\section{Dexmedetomidine up-regulates P2Y12 via miR-618}

The predicted pairing of the target region binding site between miR-618 and P2Y12 wt was UAGAGUUU in the region of the 3 '-UTR (Figure 3 A). Next, BV-2 was transfected with miR-618 mimic and NC mimic, and the luciferase report assay was used to validate the specific effect of miR-618 on P2Y12 wt. miR-618 overexpression significantly inhibited the luciferase activity of the P2Y12 wt 3'-UTR compared with NC mimic overexpression (Figure 3B; $p<0.01$ ). However, no change was observed with the P2Y12 mut 3'UTR.
After LPS stimulation in BV-2 cells, the miR-618 level was significantly upregulated but P2Y12 was downregulated compared with that in the control group $(p<0.01)$. Dexmedetomidine treatment decreased miR-618 expression but increased P2Y12 expression ( $p<0.01)$ compared with that in LPS-treated cells $(p<$ 0.01). miR-618 overexpression significantly upregulated miR-618 expression but downregulated P2Y12 expression compared with that in LPS-treated cells $(p<0.01)$. However, miR-618 expression was significantly reduced but P2Y12 expression was increased after dexmedetomidine administration in miR-618treated cells (Figure $3 \mathrm{C}$ and $\mathrm{D} ; p<0.01$ ). miR618 overexpression downregulated P2Y12 expression in LPS-treated cells, while dexmedetomidine upregulated P2Y12 expression (Figure $3 \mathrm{E} ; p<0.01$ ).
A

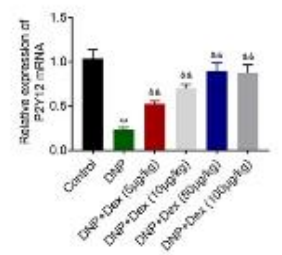

C

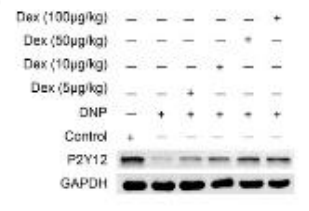

B
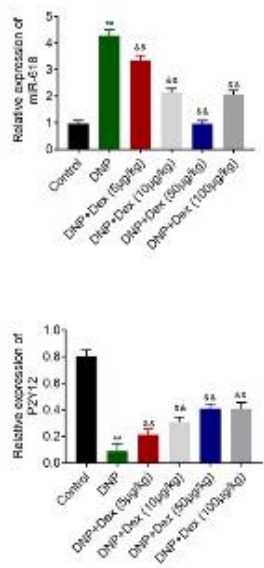

Figure 2: Dexmedetomidine inhibits miR-618 expression and increases P2Y12 expression in the spinal cord of DNP rats. A, The mRNA level of P2Y12 was determined by qRT-PCR. B, The mRNA level of miR-618 was determined by qRT-PCR. C, The protein level of P2Y12 was determined by western blotting. ${ }^{* \star} P$ $<0.01$, compared with the control group; \&\& $p<0.01$, compared with the LPS group. DEX, dexmedetomidine; DNP, diabetic neuropathic pain

\section{Dexmedetomidine inhibits microglial activation by regulating P2Y12}

LPS stimulation significantly downregulated the level of P2Y12, but the expression of IBA-1 was upregulated compared with those in the control group (Figure 4; $p<0.01$ ). Dexmedetomidine administration elevated $\mathrm{P} 2 \mathrm{Y} 12$ expression but decreased IBA-1 expression compared with LPStreated cells $(p<0.01)$. ShP2Y12, an shRNA that knocks down P2Y12, reduced P2Y12 expression, but IBA-1 expression was increased compared with that in LPS-treated cells. 
However, dexmedetomidine application upregulated P2Y12 expression but downregulated IBA-1 expression $(p<0.01)$.
A

C

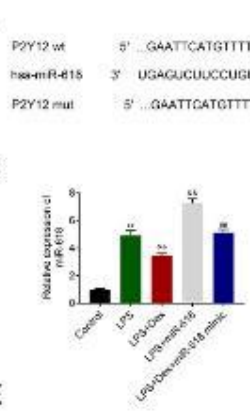

E

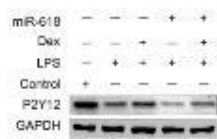

B

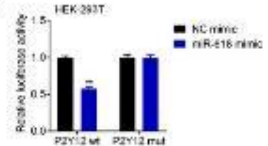

D
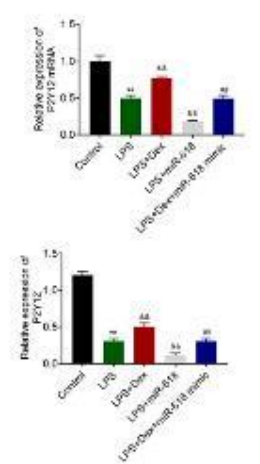

Figure 3: Dexmedetomidine up-regulates $P 2 Y 12$ via miR-618. A: Sequences of miR-618 and presumed binding site in the 3'-UTR of P2Y12. B: The luciferase reporter assay was performed after the transfection of miR-618 mimic and NC mimic into BV-2 cells C, Changes in the miR-618 mRNA levels in LPS-treated cells after dexmedetomidine and miR-618 treatment. $\mathrm{D}$ : Changes in the P2Y12 mRNA levels in LPS-treated cells after dexmedetomidine and miR-618 treatment. E: Protein levels of P2Y12 in LPS-treated cells after dexmedetomidine and miR-618 treatment. ${ }^{* *} P<0.01$, compared with the control group; ${ }^{2} p<0.01$, compared with the LPS group; ${ }^{\prime \prime} p<0.01$, compared with miR-618-treated cells. DEX, dexmedetomidine; DNP, diabetic neuropathic pain
A

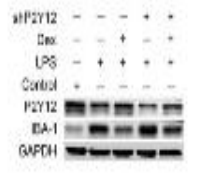

B

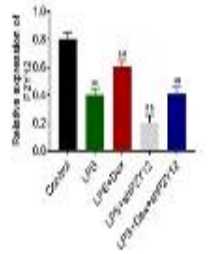

C

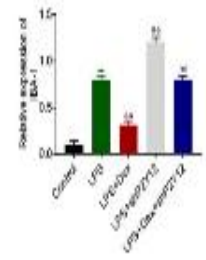

Figure 4: Dexmedetomidine inhibits microglial activation by regulating $\mathrm{P} 2 \mathrm{Y} 12$. A, P2Y12 protein expression was determined by western blot analysis. B: Protein levels of P2Y12 in LPS-treated cells after dexmedetomidine and shP2Y12 treatment. C: Protein levels of IBA-1 in LPS-treated cells after dexmedetomidine and shP2Y12 treatment. ${ }^{* *} P<0.01$, compared with the control group; ${ }^{\& \&} p<0.01$, compared with the LPS group; ${ }^{\prime} p<0.01$, compared with the shP2Y12-treated cells. DEX, dexmedetomidine; DNP, diabetic neuropathic pain; IBA-1, ionized calcium binding adaptor molecule 1

\section{DISCUSSION}

DNP is a common complication of diabetic patients, and the main manifestations are spontaneous pain, paresthesia, dysesthesia, hyperalgesia, and allodynia [15]. Duloxetine is frequently used to treat DNP clinically, but side effects such as nausea, dry mouth, constipation, fatigue, and somnolence occur in patients who receive duloxetine at a rate $>5 \%$ [16]. Thus, developing more effective and safer drugs to improve DNP treatment is warranted. In the current study, dexmedetomidine treatment significantly alleviated neuropathic pain in DNP rats and decreased miR-618 expression, but the level of P2Y12 was increased in the spinal cord of DNP rats. Dexmedetomidine inhibited microglial activation by regulating miR-618 through directly targeting P2Y12.

In many neurological diseases, the pathogenesis of microglia is complicated and microglial activation is an important response factor to pathological changes in the central nervous system [17]. Activated microglia contribute to neuropathic pain regarding sensory and/or nonsensory aspects [18]. Thus, developing new drugs targeting microglia may provide a potential therapy for neuropathic pain. The Chinese medicine $\mathrm{Wu}$-Tou decoction was demonstrated to diminish neuropathic pain through the suppression of hippocampal microglia activation [19].

Zhang et al revealed that ammoxetine relieved DNP symptoms by inhibiting microglial activation and inflammation in the spinal cord via regulating the phosphorylation of p38 and phosphorylation of c-Jun N-terminal kinase pathways [20]. In this study, increased IBA-1 expression was significantly observed after LPS stimulation in BV-2 cells, suggesting that LPS stimulated microglial activation. After dexmedetomidine treatment, microglial activity was significantly inhibited, indicating that dexmedetomidine attenuated neuropathic pain in DNP rats by inhibiting microglial activity.

MiRNAs, key modulators of differentiation, activation, and polarization of microglia and macrophages, play pivotal roles in microglia and astrocyte-mediated inflammation and neuropathic pain $[21,22]$. For example, miR190a-5p contributes to DNP through targeting SLC17A6 [23], miR-32-5p promotes neuropathic pain and neuroinflammation in rats after spinal nerve ligation by downregulating dual-specificity phosphatase 5 [24]. In the present study, STZ injection and LPS stimulation induced significant increases in miR-618, while dexmedetomidine 
administration decreased miR-618 expression in DNP rats and LPS-treated BV-2 cells.

To investigate the mechanism by which dexmedetomidine decreased miR-618 expression in the spinal cord of DNP rats, the dual luciferase reporter assay was performed. The findings revealed the paired sequence of miR-618 located in the 3'-UTR of P2Y12 wt, implying the direct interaction between miR-618 and the 3'-UTRs of P2Y12. As one type of microglial purinergic receptor, P2Y12 is involved in the pathogenesis of neuropathic pain [25]. A recent study found that the microglial P2Y12 receptor regulated microglial activation and was considered a therapeutic remedy to alleviate neuropathic pain [26]. In this study, STZ injection and LPS stimulation decreased P2Y12 expression. Dexmedetomidine significantly elevated P2Y12 expression, but miR-618 inhibited P2Y12 expression. The results suggest that dexmedetomidine inhibits microglial activation by regulating miR-618 through directly targeting P2Y12.

\section{CONCLUSION}

This study demonstrates that dexmedetomidine attenuates diabetic neuropathic pain by inhibiting microglial activation via the regulation of the miR618/P2Y12 pathway, suggesting that miR-618 might provide a novel clinical therapeutic strategy for patients with DNP.

\section{DECLARATIONS}

\section{Acknowledgement}

This work was supported by the Inner Mongolia Natural Science Foundation (Grant no. 2019MS08186).

\section{Competing interests}

The authors state that there are no conflicts of interest to disclose regarding this work.

\section{Contribution of authors}

We declare that this work was performed by the authors named in this article and all liabilities pertaining to claims relating to the content of this article will be borne by the authors. Jiannan Song designed the study, supervised the data collection. Shan Cong analyzed and interpreted the data. Yan Qiao prepared the manuscript for publication and reviewed the draft of the manuscript. All the authors have read and approved the manuscript.

\section{Open Access}

This is an Open Access article that uses a funding model which does not charge readers or their institutions for access and distributed under the terms of the Creative Commons Attribution License (http://creativecommons.org/licenses/by/ 4.0) and the Budapest Open Access Initiative (http://www.budapestopenaccessinitiative.org/rea d), which permit unrestricted use, distribution, and reproduction in any medium, provided the original work is properly credited.

\section{REFERENCES}

1. Liao C, Zhang $W$, Yang M, Ma Q, Li G, Zhong W. Surgical Decompression of Painful Diabetic Peripheral Neuropathy: The Role of Pain Distribution. PloS One 2014; 9(10).

2. Abbott CA, Malik RA, Van Ross ERE, Kulkarni J, Boulton AJM. Prevalence and Characteristics of Painful Diabetic Neuropathy in a Large Community-Based Diabetic Population in the U.K. Diabetes Care 2011; 34(10): 2220-2224.

3. Wang $D$, Couture R, Hong Y. Activated microglia in the spinal cord underlies diabetic neuropathic pain. European Journal of Pharmacology 2014; 728(59-66.

4. Mantz J, Josserand J, Hamada S. Dexmedetomidine: new insights. European Journal of Anaesthesiology 2011; 28(1): 3-6.

5. Qiu Z, Lu P, Wang K, Zhao X, Li Q, Wen J, Zhang H, Li $R$, Wei $H, \quad L V \quad Y$. Dexmedetomidine Inhibits Neuroinflammation by Altering Microglial M1/M2 Polarization Through MAPK/ERK Pathway. Neurochemical Research 2020; 45(2): 345-353.

6. Zhou T, Wu J, Chen Z, Liu Z, Miao B. Effects of dexmedetomidine on P2X4Rs, p38-MAPK and BDNF in spinal microglia in rats with spared nerve injury. Brain Research 2014; 1568(21-30.

7. Zhong J, Lu Y, Zhang J. Dexmedetomidine Reduces Diabetic Neuropathy Pain in Rats through the Wnt 10a/ß-Catenin Signaling Pathway. BioMed Research International 2018; 2018(9043628.

8. Shi D, Yuan Y, Ye D, Kang L, Wen J, Chen H. MiR-1835p Alleviates Chronic Constriction Injury-Induced Neuropathic Pain Through Inhibition of TREK-1. Neurochemical Research 2018; 43(6): 1143-1149.

9. Zuo J, Luo R, Huang C, Lou X, Li L. MiR-126 enhances cisplatin chemosensitivity in hepatocellular carcinoma cells by targeting IRS1. Tropical Journal of Pharmaceutical Research 2019; 18(1): 25-30.

10. Wang WW, Yang L, Wu J, Gao C, Zhu YX, Zhang $D$, Zhang $H X$. The function of miR-218 and miR-618 in postmenopausal osteoporosis. European review for medical and pharmacological sciences 2017; 21(24): 5534.

11. Shi J, Gong L, Chen L, Luo J, Song G, Xu J, Lv Z, Tao H, Xia $Y, Y e$ Z. miR-618 Suppresses Metastasis in Gastric

Trop J Pharm Res, January 2021; 20(1): 66 
Cancer by Downregulating the Expression of TGF- $\beta 2$. Anatomical Record-advances in Integrative Anatomy and Evolutionary Biology 2019; 302(6): 931-940.

12. Santosbezerra DP, Santos AS, Guimaraes GC, Admoni SN, Perez RV, Machado CG, Pelaes TS, Passarelli M, Machado UF, Queiroz MS. Micro-RNAs 518d-3p and 618 Are Upregulated in Individuals with Type 1 Diabetes with Multiple Microvascular Complications. Frontiers in Endocrinology 2019; 10 (

13. Care IoLARCo, Animals UoL: Guide for the care and use of laboratory animals: US Department of Health and Human Services, Public Health Service, National ...; 1986.

14. Livak KJ, Schmittgen TD. Analysis of relative gene expression data using real-time quantitative PCR and the 2(-Delta Delta C(T)) Method. Methods 2001; 25(4): 402-408.

15. Van Hecke O, Austin SK, Khan RA, Smith BH, Torrance $N$. Neuropathic pain in the general population: $A$ systematic review of epidemiological studies. Pain 2014; 155(4): 654-662.

16. Haddad PM. Safety and tolerability of duloxetine adverse events and how to handle them. Annals of General Psychiatry 2006; 5(1): 1-1.

17. Kreutzberg GW. Microglia: a sensor for pathological events in the CNS. Trends in Neurosciences 1996; 19(8): 312-318.

18. Inoue K, Tsuda M. Microglia in neuropathic pain: cellular and molecular mechanisms and therapeutic potential. Nature Reviews Neuroscience 2018; 19(3): 138-152.

19. Zhu C, Xu Q, Mao Z, Lin N. The Chinese Medicine WuTou Decoction Relieves Neuropathic Pain by Inhibiting
Hippocampal Microglia Activation. Scientific Reports 2018; 8(1): 12292.

20. Zhang T, Xue R, Fan S, Fan Q, An L, Li J, Zhu L, Ran Y, Zhang L, Zhong B. Ammoxetine attenuates diabetic neuropathic pain through inhibiting microglial activation and neuroinflammation in the spinal cord. Journal of Neuroinflammation 2018; 15(1): 176.

21. Andersen HH, Duroux M, Gazerani P. MicroRNAs as modulators and biomarkers of inflammatory and neuropathic pain conditions. Neurobiology of Disease 2014; 71(159-168.

22. Ponomarev ED, Veremeyko T, Weiner HL. MicroRNAs are Universal Regulators of Differentiation, Activation, and Polarization of Microglia and Macrophages in Normal and Diseased CNS. Glia 2013; 61(1): 91-103.

23. Yang $D$, Yang $Q$, Wei X, Liu Y, Ma D, Li J, Wan Y, Luo Y. The role of miR-190a-5p contributes to diabetic neuropathic pain via targeting SLC17A6. Journal of Pain Research 2017; 10(2395-2403.

24. Adameova AD, Bhullar SK, Elimban V, Dhalla NS. Activation of $\beta$ 1-adrenoceptors may not be involved in arrhythmogenesis in ischemic heart disease. Reviews in Cardiovascular Medicine 2018; 19(3).

25. Trang T, Beggs S, Salter MW. ATP receptors gate microglia signaling in neuropathic pain. Experimental Neurology 2012; 234(2): 354-361.

26. Gu N, Eyo UB, Murugan M, Peng J, Matta S, Dong H, Wu L. Microglial P2Y12 Receptors Regulate Microglial Activation and Surveillance during Neuropathic Pain. Brain Behavior and Immunity 2016; 55(82-92. 